\title{
Contrast between Prevalence of HIT Antibodies and Confirmed HIT in Hospitalized COVID-19 Patients: A Prospective Study with Clinical Implications
}

\author{
Maxime Delrue ${ }^{1,2}$ Virginie Siguret ${ }^{1,3}$ Marie Neuwirth ${ }^{1,3}$ Caren Brumpt ${ }^{1}$ Sebastian Voicu ${ }^{4,5}$ \\ Ruxandra Burlacu $^{6}$ Damien Sène ${ }^{6}$ Benjamin G. Chousterman ${ }^{7,8}$ Nassim Mohamedi ${ }^{9}$ \\ Thomas Lecompte ${ }^{10,11}$ Bruno Mégarbane ${ }^{4,5}$ Alain Stépanian ${ }^{1,2}$
}

\footnotetext{
${ }^{1}$ Hematology Laboratory, AP-HP Lariboisière Hospital, Paris University, Paris, France

2 EA 3518, Paris University, Paris, France

3 INSERM UMRS 1140, Paris University, Paris, France

${ }^{4}$ Department of Medical and Toxicological Critical Care, AP-HP

Lariboisière Hospital, Paris University, Paris, France

${ }^{5}$ INSERM UMRS 1144, Paris University, Paris, France

${ }^{6}$ Department of Internal Medicine, AP-HP Lariboisière Hospital, Paris University, Paris, France

${ }^{7}$ Department of Anesthesiology and Critical Care, AP-HP Lariboisière Hospital, Paris University, Paris, France

8 INSERM UMRS 942, Université de Paris, Paris, France

${ }^{9}$ Department of Clinical Physiology, AP-HP Lariboisière Hospital, Paris University, Paris, France

10 Département de Médecine, Service d'Angiologie et d'Hémostase, Hôpitaux Universitaires de Genève, Genève, Switzerland

11 Faculté de Médecine Geneva Platelet Group (GpG), Université de Genève, Geneva, Switzerland
}

Thromb Haemost 2021;121:971-975.

In hospitalized severe acute respiratory syndrome coronavirus-2 (SARS-CoV-2)-infected patients, elevated prevalence of thromboembolic events (TE) has been reported with subsequent recommendations to reinforce prophylactic anticoagulation using low-molecular-weight heparin $(\mathrm{LMWH})$ or unfractionated heparin (UFH). ${ }^{1}$ Heparin-induced thrombocytopenia (HIT) is an immune-mediated prothrombotic disorder resulting from immunoglobulin $G(\operatorname{IgG})$ platelet-activating antibodies against platelet chemokines, mainly platelet factor-4 (PF4), bound to heparin (HIT-associated antibodies). ${ }^{2}$ Due to SARS-CoV-2-mediated exacerbated inflammatory and procoagulant response, patients may be subject to HIT. To date, HIT data in SARS-CoV-2-infected patients are scarce, limited to the report of a few HIT cases confirmed using platelet activation assay, and HIT-associated antibody prevalence remains poorly investigated. ${ }^{3-13}$ Our prospective cohort study aimed to characterize patients with clinical suspicion of HIT and determine HIT-associated antibody prevalence in hospitalized SARS-CoV-2-infected patients. This study was part of the

received

November 27, 2020

accepted

November 30, 2020

published online

December 9, 2020

Address for correspondence Alain Stépanian, PharmD, PhD, AP-HP Hôpital Lariboisière, Service d'Hématologie biologique, 2 rue

Ambroise Paré, 75010 Paris, France (e-mail: alain.stepanian@aphp.fr).

ICU-COVID and French-COVID cohort registries approved by our institutional ethics committee (IDRCB, 2020-A00256-33; CPP, 11-202020.02.04.68737).

All consecutive SARS-CoV-2-infected adults admitted from March 2, 2020 to May 7, 2020 to the intensive care unit (ICU) and medical wards were included. Lower limb deep vein thrombosis (DVT) was diagnosed using duplex ultrasound performed weekly for critically ill patients and based on clinical suspicion for noncritically ill patients. Suspected pulmonary embolism was confirmed using chest computed-tomography/angiography.

For each patient referred by the attending physician for HIT suspicion, we systematically performed (1) 4Ts-score ${ }^{2}$; (2) qualitative particle gel immunoassay (PaGIA) (ID-PaGIA Heparin/PF4-Antibody Test, Bio-Rad, United States); (3) enzyme-linked immunosorbent assay (EIA; ZYMUTEST-HIAIgG, HYPHEN BioMed, France) with 0.500 optical density (OD) as a positivity threshold; and (4) heparin-induced platelet activation-assay (HIPLA) with a positivity threshold of $13 \%$

(c) 2020. Thieme. All rights reserved. Georg Thieme Verlag KG,

Rüdigerstraße 14,

70469 Stuttgart, Germany
DOI https://doi.org/

10.1055/a-1333-4688. ISSN 0340-6245. 


\begin{tabular}{|c|c|c|c|c|c|c|c|c|c|c|c|c|c|c|}
\hline 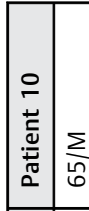 & 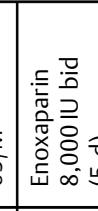 & 2 & 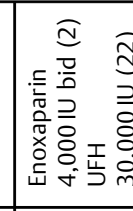 & 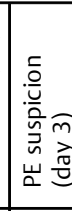 & $\frac{\bar{c}}{\frac{1}{m}}$ & 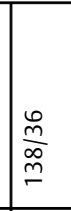 & $\stackrel{\Delta}{\Delta}$ & 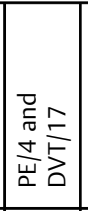 & & 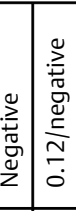 & $\curvearrowright$ & $\underline{z}$ & 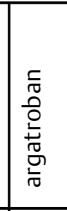 & \\
\hline 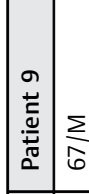 & $\stackrel{2}{2}$ & z & 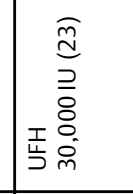 & 荌 & 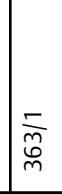 & $\begin{array}{l}\frac{d}{\infty} \\
\stackrel{\infty}{\infty} \\
\end{array}$ & 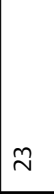 & 咅 & 0 & 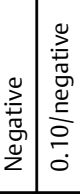 & $\underline{z}$ & $\hat{z}$ & 竞 & \\
\hline in is & & $\stackrel{2}{z}$ & 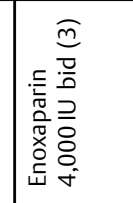 & 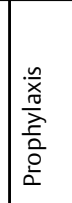 & 5 & $\mid \begin{array}{c}\frac{N}{0} \\
\\
\end{array}$ & $\simeq$ & 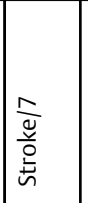 & & 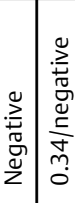 & & $\hat{z}$ & $\frac{\sigma_{0}^{\circ}}{2}$ & \\
\hline
\end{tabular}

离 
(Emo-test HIT-CONFIRM, EMOSIS-SAS, France).$^{14}$ Serotonin release assay (SRA; positivity threshold of $30 \% ; 0.1,0.5$, and $10 \mathrm{IU} / \mathrm{mL}$ heparin ), considered as the gold standard to confirm HIT diagnosis, was performed if HIT was highly likely. ${ }^{2}$

Additionally, in an exploratory study, we screened HITassociated antibodies using EIA with both ZYMUTEST-HIAIgG and ZYMUTEST-HIA-IgGAM (HYPHEN BioMed, France) in consecutive patients admitted from March 17, 2020 to April 21, 2020.

Quantitative variables are expressed as medians [25th75th percentiles] and categorical variables as percentages. Mann-Whitney and Fisher's exact tests were used for comparisons as appropriate. Pearson correlation coefficients were determined. $p<0.05$ was considered as significant.

From March 2, 2020 to May 7, 2020, 626 SARS-CoV-2infected patients were admitted, 184 patients to the ICU and 442 to the medical ward. HIT was clinically suspected in 10 patients and confirmed in one ( - Table 1 ). Considering that all patients were exposed to heparin, HIT prevalence was 1.6/1,000 patients. All patients with suspected HIT were in the ICU and had received UFH (except for one patient). Patient 6 (body mass index: $30 \mathrm{~kg} / \mathrm{m}^{2}$ ) developed cholecystitis complicated by renal failure, requiring cholecystectomy. On HIT suspicion associated with confirmed DVT, he received argatroban during 13 days (initial dose: $0.5 \mu \mathrm{kg}^{-1} \mathrm{~min}^{-1}$ ). Meanwhile, the platelet count increased on day $3(89 \mathrm{G} / \mathrm{L})$ and normalized on day 7 (264G/L). Danaparoid (1,250 IU/ $12 \mathrm{~h}$ ) was further administered for 19 days, once the renal function was normalized. HIT diagnosis was confirmed with positive SRA. He was discharged with $5 \mathrm{mg}$ bid apixaban. In the nine other patients with HIT suspicion, platelet counts declined within 6 to 36 days of heparin exposure; however, HIT-associated antibodies were undetectable with PaGIA, anti-IgG EIA (OD: 0.100-0.340), and functional HIPLA $(<13 \%$, except patient 3$)$.

Using EIA in 172 consecutive SARS-CoV-2-infected patients including 64 ICU and 108 noncritically ill patients admitted from March 17, 2020 to April 21, 2020 (-Table 2), we observed an overall $33 \%$ prevalence of anti-IgG/A/M and $11 \%$ anti-IgG HIT-associated antibodies (all positive for anti$\operatorname{IgG} / \mathrm{A} / \mathrm{M})$, without significant differences in relation to the hospitalization site. These patients have received LMWH (enoxaparin) in $87 \%$ of cases. TE compared with non-TE patients exhibited no difference between HIT-associated IgG/A/M antibody titers (OD: 0.401 [0.211-0.672] vs. 0.328 [0.217-0.534], $p=0.11$ ) or between HIT-associated IgG/A/M antibody proportions with OD $>1.0(17$ vs. $9 \%, p=0.4)$. Moreover, HIT-associated IgG/A/M antibodies (OD: 0.344 [0.218-0.550] vs. $0.318[0.206-0.686], p=0.69)$ and the proportion of HIT-associated $\mathrm{IgG} / \mathrm{A} / \mathrm{M}$ antibodies with $\mathrm{OD}$ $>1.0$ (20 vs. $10 \%, p=0.09$ ) did not significantly differ between survivors and nonsurvivors. In 11 samples with the highest HIT-associated $\mathrm{IgG} / \mathrm{A} / \mathrm{M}$ antibodies (OD range: 1.020-4.500; five also positive for IgG), HIPLA was weakly positive in one patient only (14\%; OD: 4.500 ) and negative in all others. None of the patients received HIT diagnosis. HITassociated $\operatorname{Ig} G / A / M$ antibody titers were correlated neither
Table 2 Clinical characteristics and laboratory data in the 172 consecutive COVID-19a patients screened for HIT-associated antibodies (EIA)

\begin{tabular}{|c|c|}
\hline \multicolumn{2}{|l|}{ Demographics and past medical history } \\
\hline Female/male gender & $53 / 119(31 \% / 69 \%)$ \\
\hline Age, y & $68[58-77]$ \\
\hline Body-mass index, $\mathrm{kg} / \mathrm{m}^{2}$ & $27[24-31]$ \\
\hline Diabetes & $76(45 \%)$ \\
\hline Ischemic heart disease & $44(26 \%)$ \\
\hline Autoimmune disease & $8(5 \%)$ \\
\hline \multicolumn{2}{|c|}{ Severity of disease and outcomes during hospitalization stay } \\
\hline Critically ill patients & $64(37 \%)$ \\
\hline $\mathrm{PaO}_{2} / \mathrm{FiO}_{2}, \mathrm{mmHg}(\mathrm{N}=64)^{\mathrm{b}}$ & $144[95-142]$ \\
\hline $\begin{array}{l}\text { Maximal oxygen flow, } \\
\text { L/min }(N=108)^{c}\end{array}$ & $3[1-9]$ \\
\hline Thrombotic events & $41(25 \%)$ \\
\hline - Isolated deep venous thrombosis & 24 \\
\hline - Isolated pulmonary embolism & 11 \\
\hline $\begin{array}{l}\text { - Deep venous thrombosis and } \\
\text { pulmonary embolism }\end{array}$ & 5 \\
\hline - Stroke & 1 \\
\hline - Death & $25(15 \%)$ \\
\hline \multicolumn{2}{|c|}{ Anticoagulant treatment at the time of sampling } \\
\hline \multicolumn{2}{|l|}{ Heparin exposure $(N=172)$} \\
\hline Unfractionated heparin & $23(13 \%)$ \\
\hline - Prophylactic dose & 9 \\
\hline - Therapeutic dose & 14 \\
\hline $\begin{array}{l}\text { Low-molecular-weight heparin } \\
\text { (enoxaparin) }\end{array}$ & $149(87 \%)$ \\
\hline $\begin{array}{l}\text { - Prophylactic dose (standard or } \\
\text { reinforced) }\end{array}$ & 105 \\
\hline - Therapeutic dose & 44 \\
\hline $\begin{array}{l}\text { Median time of blood sampling } \\
\text { postadmission }(N=172)\end{array}$ & $4[3-8]$ \\
\hline \multicolumn{2}{|c|}{ Laboratory parameters, units (reference interval) } \\
\hline Leukocytes, G/L (4.0-10.0) & $7.8[5.6-10.4]$ \\
\hline $\begin{array}{l}\text { Hemoglobin, } \mathrm{g} / \mathrm{dL}(\mathrm{N}, 13.0-17.0 \text { in } \\
\text { males; } 12.0-16.0 \text { in females) }\end{array}$ & $11.7[10.3-12.8]$ \\
\hline Platelets, G/L (150-450) & $285[190-364]$ \\
\hline $\begin{array}{l}\text { Prothrombin time, } \\
\text { ratio }(0.8-1.20)\end{array}$ & $1.08[1.03-1.16]$ \\
\hline Fibrinogen, G/L (2.0-4.0) & $6.69[5.15-8.03]$ \\
\hline D-dimers, ng/mL $(<500)$ & $1,900[805-3,275]$ \\
\hline $\begin{array}{l}\text { Serum creatinine, } \mu \mathrm{mol} / \mathrm{L}(64-104) \\
(N=167)\end{array}$ & $80[62-121]$ \\
\hline $\begin{array}{l}\text { C-reactive protein, } \mathrm{mg} / \mathrm{L} \\
(\leq 5)(N=130)\end{array}$ & $91[39-175]$ \\
\hline Antithrombin, IU/dL (80-120) & 93 [82-103] \\
\hline \multicolumn{2}{|l|}{ Anti-Xa activity, IU/mL } \\
\hline - Unfractionated heparin $(N=23)$ & $0.25[0.19-0.46]$ \\
\hline $\begin{array}{l}\text { - Low-molecular-weight heparin } \\
(N=147)\end{array}$ & $0.19[0.10-0.35]$ \\
\hline
\end{tabular}

(Continued) 
Table 2 (Continued)

\begin{tabular}{|c|c|}
\hline \multicolumn{2}{|l|}{ HIT-associated antibodies (EIA) } \\
\hline IgG/A/M antibodies, OD $(N=172)$ & $0.343[0.217-0.570]$ \\
\hline - Critically ill $(N=64)$ & $0.330[0.210-0.600]$ \\
\hline - Noncritically ill $(N=108)$ & $0.346[0.219-0.557]$ \\
\hline IgG antibodies, OD $(N=145)$ & $0.104[0.057-0.202]$ \\
\hline - Critically ill $(N=50)$ & $0.098[0.053-0.185]$ \\
\hline - Noncritically ill $(N=95)$ & $0.120[0.070-0.230]$ \\
\hline $\begin{array}{l}\text { Positive HIT-associated IgG/A/M antibod- } \\
\text { ies }(>0.500 \text { OD) }\end{array}$ & $57(33 \%)$ \\
\hline - Critically ill patients & 21 \\
\hline - Noncritically ill patients & 36 \\
\hline $\begin{array}{l}\text { Positive HIT-associated IgG antibodies } \\
(>0.500 \text { OD) }\end{array}$ & $16(11 \%)$ \\
\hline - Critically ill patients & 6 \\
\hline - Noncritically ill patients & 10 \\
\hline $\begin{array}{l}\text { HIT-associated } \lg \mathrm{G} / \mathrm{A} / \mathrm{M} \text { antibodies with } \\
\mathrm{OD}>1.0\end{array}$ & $19(11 \%)$ \\
\hline \multicolumn{2}{|l|}{ In relation to thromboembolic events (TE) } \\
\hline - Patients with TE $(N=41)$ & $7(17 \%)$ \\
\hline - Patients without TE $(N=131)$ & $12(9 \%)$ \\
\hline \multicolumn{2}{|l|}{ In relation to outcome } \\
\hline - Nonsurvivors $(N=25)$ & $5(20 \%)$ \\
\hline - Survivors $(N=147)$ & $14(10 \%)$ \\
\hline
\end{tabular}

Abbreviations: HIT, heparin-induced thrombocytopenia; IgG, immunoglobulin G; OD, optical density.

Note: data are expressed as median [25th-75th percentiles] or numbers (percentages) as appropriate. Five COVID-19 patients required extracorporeal membrane oxygenation, with the following $\mathrm{IgG} / \mathrm{A} / \mathrm{M}$ and $\mathrm{IgC}$ HIT-antibody titers: (0.812 and 1.125), (1.703 and 0.097), (0.297 and $0.092),(0.400$ and 0.056$)$, and (0.484 and 0.143$)$, respectively.

aSARS-CoV-2 infection was diagnosed on admission using RT-PCR (Cobas-SARS-CoV-2 kits, Roche, France).

${ }^{\mathrm{b}}$ In the ICU patients.

cIn the medical ward patients.

with fibrinogen $(p=0.09)$, nor with C-reactive protein $(p=0.11)$

In addition to the HIT prevalence, we provided new data on the overall HIT-associated antibody prevalence in hospitalized SARS-CoV-2-infected patients, predominantly receiving LMWH. The 1.6/1,000 patient prevalence of HIT is closer to values previously reported in critically ill patients $(0.20-0.45 \%)$ than to values in cardiac surgery patients $(\sim 1-3 \%)^{5}$ and lower than the estimated overall HIT incidence $(0.76 \%) .{ }^{6}$ Patell et al reported a cumulative $12 \%$ incidence of positive antibodies in hospitalized COVID-19 patients using an immunoassay, with one confirmed HIT case. ${ }^{6}$ Daviet et al confirmed HIT diagnosis in seven out of 86 ICU COVID-19 patients. ${ }^{9}$ The lower frequency of confirmed HIT in our ICU patients could be partly explained by the preferential use of LWMH (in 70\% of our ICU patients) rather than UFH. Upon clinical HIT suspicion, we calculated the 4Ts-score and whatever the score probability was, we performed HIT-associated antibody immunoassays since this score had not been validated in SARS-CoV-2-infected patients. We were able to exclude HIT diagnosis in nine patients. In addition, we systematically performed a supplemental functional assay (HIPLA), which turned out to be negative, but for one patient (weakly positive). Noteworthy, in our series of patients referred for HIT suspicion, non-HIT-related thrombocytopenia/thrombosis was associated with a high risk for fatal outcome, mostly in the setting of multiorgan failure. Conversely, we found an elevated frequency of HIT-associated $\mathrm{IgG} / \mathrm{A} / \mathrm{M}$ antibodies in hospitalized SARS-CoV-2-infected patients, consistent with the highest seroconversion rates observed in cardiac surgery patients assisted with extracorporeal membrane oxygenation or ventricular assist device ( $25-75 \%)$. These antibodies seem to be nonfunctional since the platelet activation assay (HIPLA) was essentially negative in the patients with the highest EIA OD $(N=11)$. However, HIT-associated IgG antibody frequency was as expected lower, similar to seroconversion rates observed among medical and surgical patients ( 4-17\%).

Limitations of the current study include the single-center setting and the short study period.

To conclude, our data suggest that COVID-19 patients receiving $\mathrm{LMWH}$ do not appear to be especially susceptible to HIT. Prevalence of HIT-associated antibodies is comparable to other critical illness settings and those antibodies do not seem to be associated with increased risk of TE and death.

Conflict of Interest

None declared.

\section{Acknowledgments}

The case investigations, analysis, and manuscript preparation were completed as part of the official duties at the university hospital. We would like to thank Pr. Dominique Helley (Hematology Laboratory, Hôpital Européen Georges Pompidou, AP-HP, Paris, France) for serotonin release assay. We would like to acknowledge the APHP Lariboisière COVID Group for the management of COVID-19 patients admitted in our hospital.

\section{References}

1 Moores LK, Tritschler T, Brosnahan S, et al. Prevention, diagnosis, and treatment of VTE in patients with coronavirus disease 2019: CHEST guideline and expert panel report. Chest 2020;158(03): 1143-1163

2 Warkentin TE. Laboratory diagnosis of heparin-induced thrombocytopenia. Int J Lab Hematol 2019;41(Suppl 1):15-25

3 Lingamaneni P, Gonakoti S, Moturi K, Vohra I, Zia M. Heparininduced thrombocytopenia in COVID-19. J Investig Med High Impact Case Rep 2020. Doi: 10.1177/2324709620944091

4 Dragonetti D, Guarini G, Pizzuti M. Detection of anti-heparin-PF4 complex antibodies in COVID-19 patients on heparin therapy. Blood Transfus 2020;18(04):328

5 Riker RR, May TL, Fraser GL, et al. Heparin-induced thrombocytopenia with thrombosis in COVID-19 adult respiratory distress syndrome. Res Pract Thromb Haemost 2020;4(05):936-941

6 Patell R, Khan AM, Bogue T, et al. Heparin induced thrombocytopenia antibodies in Covid-19. Am J Hematol 2020; 95(10): E295-E296

7 Bidar F, Hékimian G, Martin-Toutain I, Lebreton G, Combes A, Frère C. Heparin-induced thrombocytopenia in COVID-19 patients with severe acute respiratory distress syndrome requiring extracorporeal membrane oxygenation: two case reports. J Artif Organs 2020. Doi: $10.1007 / s 10047-020-01203-\mathrm{x}$ 
8 Huang CT, Hsu SY, Chang KW, Huang CG, Yang CT, Cheng MH. Heparin-induced thrombocytopenia and thrombosis in a patient with Covid-19. Thromb Res 2020;196:11-14

9 Daviet F, Guervilly C, Baldesi O, et al. Heparin-induced thrombocytopenia in severe COVID-19. Circulation 2020;142(19):1875-1877

10 Parzy G, Daviet F, Puech B, et al. Venous thromboembolism events following venovenous extracorporeal membrane oxygenation for severe acute respiratory syndrome coronavirus 2 based on CT scans. Crit Care Med 2020;48(10):e971-e975

11 Tran M, Sheth C, Bhandari R, Cameron SJ, Hornacek D. SARS-CoV-2 and pulmonary embolism: who stole the platelets? Thromb J 2020;18:16
12 Ogawa Y, Nagata T, Akiyama T, et al. Argatroban therapy for heparin-induced thrombocytopenia in a patient with coronavirus disease 2019. J Thromb Thrombolysis 2020;50(04): 1012-1014

13 May JE, Siniard RC, Marques M. The challenges of diagnosing heparin-induced thrombocytopenia in patients with COVID-19. Res Pract Thromb Haemost 2020;4(06):1066-1067

14 Vayne C, Guéry EA, Charuel N, et al. Evaluation of functional assays for the diagnosis of heparin induced thrombocytopenia using 5B9, a monoclonal IgG that mimics human antibodies. J Thromb Haemost 2020;18(04):968-975 